\title{
Discovery of the antigraviton verified by the rotation curve of NGC 6503
}

\author{
Wing-Hong Wong(黃穎航)*, Wing-To Wong (黃穎濤),Wing-Keung Wong (黃穎強), Ling-Mei Wong (黃靈美) \\ China Evangelical Seminary, Taipei City, Taiwan \\ *Corresponding author E-mail: jwhwong@ces.org.tw
}

\begin{abstract}
Using special relativity theory, it was discovered that to each graviton, there coexists its antiparticle, an antigraviton. Building on this discovery and quantum theory, a new quantum gravity theory (QGT) was invented, providing equations of the gravitational potential, the graviton wavelength, and the circular speed, without the dark matter hypothesis (DMH). According to this QGT, the gravitational scalelength of a spiral galaxy depends on its mass distribution, being approximately equal to 1.5708 times the radius of its "radial center of mass". Also Newtonian gravity theory (NGT) is of limited validity: if the radius is less than the gravitational scale-length, NGT is exact; but if the radius is greater than the gravitational scale-length, it becomes inadequate, and is surpassed by QGT. When the theoretical rotation curve was compared with the rotation curve of the galaxy NGC6503, the QGT was verified within the rms error of the measured rotation speeds, 3.0 percent. It was also demonstrated how two formulas of general relativity theory (Schwarzschild metric, gravitational time dilation) may be revised by the QGT, and thus be upgraded to formulas of quantum general relativity theory. It is suggested the socalled "dark matter" problem can be solved by discarding the DMH and adopting our new QGT.
\end{abstract}

Keywords: Cosmology: Theory, Dark Matter, Galaxies: Kinematics and Dynamics, Galaxies: Individual (NGC6503), Gravitation, Large-Scale Structure of Universe.

\section{Introduction}

Before embarking on graviton physics, let us recall briefly the dark matter problem. In the early 1970s, the astronomer Rubin first adopted the concept of dark (non-luminous) matter in explaining the shape of the rotation curve of a typical spiral galaxy In the late 1970s, more astronomers accepted the dark matter hypothesis in galaxy dynamics. They noticed that a typical mass model of a spiral galaxy, which assumes a fixed mass-to-light ratio, is only able to represent the observed rotation curve in the inner region, but fails in the outer region. When the radius $R$ is large enough to contain nearly all the galactic mass, the orbital speed does not decrease in the Newtonian-Keplerian way (inversely as the square root of $R$ ), but remains roughly constant. By the 1980s, most astronomers interpreted this puzzling discrepancy in the outer region as an evidence for the presence of dark matter in galaxies (Rubin 1983, Van Albada et al. 1985, Van Albada \& Sancisi 1986, Kent 1987); but this mysterious discrepancy is still an unsolved problem. In the following, it will be shown that the dark matter problem can be solved by applying the two pillars of modern physics (relativity theory and quantum theory) plus a little innovative insight or discovery.

Today it is commonly believed that the graviton (the boson which mediates the gravitational field) does not have an antiparticle, being similar to the photon of the electromagnetic field. In sec. 2, applying special relativity theory and symmetry consideration, it was discovered that the graviton coexists with its antiparticle, the antigraviton. From this discovery, using quantum theory, a new quantum gravity theory (QGT) was invented, deriving equations for the distance variations of the gravitational potential, the wavelength of gravitons, and the rotation speed, without adopting the DMH. According to this QGT, the gravitational scalelength $R_{\mathrm{o}}$ of a galaxy is related to its mass distribution, being equal to $0.5 \pi$ times the radius of its radial center of mass. Moreover, the validity of Newtonian gravity theory (NGT) is limited: if the radius $R \leq R_{\mathrm{o}}$, NGT is exact; but if $R>R_{\mathrm{o}}$, it becomes increasingly inaccurate, and is superseded by QGT. In sec. 3, the results of comparing the QGT with data from the spiral galaxy NGC6503 are presented. In this astronomical test, the source of the gravitons and antigravitons was the galaxy NGC6503, and the detectors were the orbiting stars. The QGT was well verified as the standard deviation of the best-fitting curve from the rotation curve of NGC6503 is within the standard error of the measured rotation speeds, 3.0 percent. In sec. 4 , seven topics are discussed: (1) the concept of negative mass; (2) the non-annihilation of the graviton by the antigraviton; (3) the finite wavelength of the graviton; (4) the non-zero rest mass of the graviton; (5) virtual mass amplification by antigravitons; (6) the Schwardzchild metric; and (7) gravitational time dilation. In sec. 5, the conclusions of this paper are drawn.

\section{Theory}

\subsection{The existence of the antigraviton}

In a locally inertial frame of reference, let us consider a boson of the gravitational field. It is traveling practically at the speed of 
light $c$ because its rest mass $m_{\mathrm{o}}$ is assumed to be extremely tiny, though non-zero. Let its energy, and momentum be $E$, and $p$ respectively. From special relativity theory (Forshaw \& Smith 2009),

$$
m_{\mathrm{o}}^{2} c^{4}=E^{2}-c^{2} p^{2}
$$

Solving for $m_{\mathrm{o}}$,

$$
m_{\mathrm{o}}= \pm \sqrt{E^{2}-p^{2} c^{2}} / c^{2}
$$

Eq. (2) may be interpreted as: if the positive solution corresponds to the rest mass of a graviton $\left(\Gamma^{+}\right)$, the negative one corresponds to that of an antigraviton $\left(\Gamma^{-}\right)$, or vice versa. Thus if the names of graviton and antigraviton are interchanged, physical reality remains invariant, i.e., $\Gamma^{+}$and $\Gamma^{-}$are particle-antiparticle symmetric partners. The prediction of negative mass by special relativity theory may seem to be a novelty or a joke, but if we believe what is mathematically possible is also physically possible, we should be bold enough to accept the idea of a negative mass or the concept of an antigraviton.

\subsection{The relative abundances of gravitons and antigravitons}

Consider a subset of gravitons $\left(\Gamma_{\mathrm{s}}^{+}\right)$and antigravitons $\left(\Gamma_{\mathrm{s}}^{-}\right)$, in which the mass of each $\Gamma^{+}$is $m$, and the mass of each $\Gamma^{-}$is $-m$. Let the de Broglie wavelength of each $\Gamma^{+}$be $\lambda$, which is related to $m$ by

$\lambda=h / \mathrm{cm}$

where $h$ is Planck's constant. In this subset, as the mass of each $\Gamma^{-}$is $-m$, from eq. (3), the de Broglie wavelength of each $\Gamma^{-}$ is $-\lambda$. Let the probabilities of $\Gamma_{\mathrm{s}}^{+}$and $\Gamma_{\mathrm{s}}^{-}$in this subset be $\eta$ and $(1-\eta)$ respectively. If the names of $\Gamma_{\mathrm{s}}^{+}$and $\Gamma_{\mathrm{s}}^{-}$are interchanged, their respective probabilities become $(1-\eta)$ and $\eta$. However, after the interchange of names, physical reality remains invariant. Hence the probabilities of $\Gamma_{\mathrm{s}}^{+}$and $\Gamma_{\mathrm{s}}^{-}$remain unchanged. Therefore, $\eta=(1-\eta)$, and $\eta=0.5$. This implies that to every $\Gamma^{+}$with mass $m$, there exists one $\Gamma^{-}$with mass $-m$. Using eq. (3), it may be deduced that to each $\Gamma^{+}$with wavelength $\lambda$, there exists one $\Gamma^{-}$with wavelength $-\lambda$. Since a set of $\Gamma_{\mathrm{s}}^{+}$and $\Gamma_{\mathrm{s}}^{-}$is a union of subsets, each with a special magnitude of wavelength, the above result can be generalized as: in any differential band of wavelengths ( $\pm \lambda, \pm \lambda \pm \mathrm{d} \lambda), \Gamma_{\mathrm{s}}^{+}$and $\Gamma_{\mathrm{s}}^{-}$are equally abundant.

The equal abundances of $\Gamma_{\mathrm{s}}^{+}$and $\Gamma_{\mathrm{s}}^{-}$points to the possibility that they are created by interacting masses in pairs. The net rest energy of such a $\Gamma^{+} \& \Gamma^{-}$pair is exactly zero $\left(=m_{\mathrm{o}} c^{2}-m_{\mathrm{o}} c^{2}\right)$, consequently all the production energy is converted into kinetic energy, resulting in maximum energy efficiency in each gravitational interaction.

\subsection{The quantum gravitational potential}

Let the wavelength of a $\Gamma^{+}$be $\lambda$. At a point $P$ which is at radius $R$ from a point mass $M$ situated at the origin $\mathrm{O}$, the Yukawa potential is (Yukawa 1935)
$\Phi_{+}(R)=-\frac{G_{\mathrm{q}} M}{R} \exp \left(\frac{-R}{\lambda}\right)$

where $G_{\mathrm{q}}$ is the quantum gravitational constant, and $\lambda$ may be regarded as the range of the potential. Because the names $\Gamma^{+}$and $\Gamma^{-}$are interchangeable, and as the $\Gamma^{+}$mediates a Yukawa potential $\Phi_{+}(R)$, the coexisting $\Gamma^{-}$, whose wavelength is $-\lambda$, should also mediate a Yukawa potential

$\Phi_{-}(R)=-\frac{G_{\mathrm{q}} M}{R} \exp \left(\frac{R}{\lambda}\right)$

which is repulsive or antigravitational.

In a galactic orbit of average radius $R$, let the expectation value of the wavelength of the $\Gamma_{\mathrm{s}}^{+}$be $\lambda_{\mathrm{A}}(R)$, and that of the $\Gamma_{\mathrm{s}}^{-}$ be $-\lambda_{\mathrm{A}}(R)$. Since the $\Gamma_{\mathrm{s}}^{+}$and $\Gamma_{\mathrm{s}}^{-}$have the same probability 0.5 , applying eq. (4) and (5), the quantum gravitational potential is

$$
\begin{gathered}
\Phi_{\mathrm{q}}(R)=-\frac{G_{\mathrm{q}} M(R)\left\{\exp \left[-R / \lambda_{\mathrm{A}}(R)\right]+\exp \left[R / \lambda_{\mathrm{A}}(R)\right]\right\}}{2 R} \\
\Phi_{\mathrm{q}}(R)=-\frac{G_{\mathrm{q}} M(R) \cosh \left[R / \lambda_{\mathrm{A}}(R)\right]}{R}=-\frac{G_{\mathrm{q}} M(R) \cosh \left[-R / \lambda_{\mathrm{A}}(R)\right]}{R}
\end{gathered}
$$

Eq. (6.1) shows that, if $\Gamma_{\mathrm{s}}^{+}$are interchanged with $\Gamma_{\mathrm{s}}^{-}$, the quantum potential $\Phi_{\mathrm{q}}(R)$ remains invariant. And eq. (6.2) shows that in evaluating $\Phi_{\mathrm{q}}(R)$, it is sufficient to consider either the wavelength of $\Gamma_{\mathrm{s}}^{+}$or $\Gamma_{\mathrm{s}}^{-}$, for the cosh function is even.

\subsection{The fundamental wavelength of the gravitons in a spiral galaxy}

For a galaxy, let us define the radius of its radial center of mass (RCM) as the ratio of the first moment about the galactic axis to the total mass of the galaxy, i.e.:

$R_{\mathrm{RCM}}=\int_{0}^{\infty} 2 \pi \rho(R) R^{2} d R / \int_{0}^{\infty} 2 \pi \rho(R) R d R$, where $\rho(R)$ is the surface density as a function of the radius $R$. Simplifying, we have

$R_{\mathrm{RCM}}=\int_{0}^{\infty} \rho(R) R^{2} d R / \int_{0}^{\infty} \rho(R) R d R$.

For a spiral galaxy, since its materials (stars and gas molecules) move in elliptical orbits, its RCM moves in an elliptical orbit in the galactic plane. Let the perimeter of the orbit of the RCM be $C_{\mathrm{RCM}}$, we have $C_{\mathrm{RCM}}=2 \pi R_{\mathrm{RCM}}$. As $C_{\mathrm{RCM}}$ is the average mean free path (mfp) of the orbiting materials, it may be equated with the mfp of the gravitons operating in the galaxy. Thus the fundamental wavelength $\lambda_{\mathrm{o}}$ of the galactic gravitons equals $C_{\mathrm{RCM}}$.

$\lambda_{\mathrm{o}}=C_{\mathrm{RCM}}=2 \pi R_{\mathrm{RCM}}$

As a corollary, denoting the quarter fundamental wavelength $\lambda_{0} / 4$

by $R_{\mathrm{o}}$, from eq. (8), we obtain

$R_{\mathrm{o}}=\lambda_{\mathrm{o}} / 4=0.5 \pi R_{\mathrm{RCM}}=1.570796 R_{\mathrm{RCM}}(7 \mathrm{sig}$. fig. $)$

\subsection{The expectation value of the graviton wavelength as a function of radius}

Let us consider the case when $R=R_{\mathrm{o}}$. Assuming that graviton waves behave like sound waves in a resonance tube of length $R$, a resonance occurs when the tube length is equal to the quarter 
wavelength $R_{\mathrm{o}}$ of the waves. Hence, if $R=R_{\mathrm{o}}$, the expectation value of the graviton wavelength is

$\lambda_{\mathrm{A}}\left(R_{\mathrm{o}}\right)=R_{\mathrm{o}}$

At the radius $R_{\mathrm{o}}$, from eq. (10), the cosh term in eq. (6.2) equals $\cosh (1.0)$, and the quantum potential reduces to the Newtonian potential. Accordingly, the Newtonian gravitational constant $G_{\mathrm{n}}$ is related to $G_{\mathrm{q}}$ by

$G_{\mathrm{n}}=G_{\mathrm{q}} \cosh (1)=1.543081 G_{\mathrm{q}}(7$ sig. fig. $)$

$G_{\mathrm{q}}=G_{\mathrm{n}} / \cosh (1)=0.6480543 G_{\mathrm{n}}(7$ sig. fig. $)$

Thus the radius $R_{\mathrm{o}}$ is within the inner region of a galaxy, where Newtonian gravity theory (NGT) is experimentally known to be exact. This implies that conversely, if $R \leq R_{\mathrm{o}}$, the quantum potential in eq. (6.2) reduces to the Newtonian potential Consequently,

$G_{\mathrm{q}} \cosh [R / \lambda(R)]=G_{\mathrm{n}}$

Eq. (12) is true only if its cosh term equals a constant, which in turn implies that

$\lambda_{\mathrm{A}}(R)=c_{1} R$

where $c_{1}$ is a constant of proportionality. From eq. (10) and (13), we have, in this NGT region

$c_{1}=1$

Substituting eq. (14) into eq. (13), we have, in this Newtonian region,

$$
\begin{aligned}
& \lambda_{\mathrm{A}}(R)=R \\
& \frac{d \lambda_{\mathrm{A}}(R)}{d R}=1
\end{aligned}
$$

In the region $R>R_{\mathrm{o}}$, let us proceed by quantum theory. Denoting the increase of $\lambda_{\mathrm{A}}(R)$ when the radius increases from $R_{\mathrm{o}}$ to $R$ by $\Delta \lambda_{\mathrm{A}}(R)$, we get

$$
\lambda_{\mathrm{A}}(R)=R_{\mathrm{o}}+\Delta \lambda_{\mathrm{A}}(R)
$$

Next let us derive an expression for the probability density of the gravitons as a function of $\lambda$, and then as a function of $R$. As gravitons are bosons, their probability density $B(E)$ at temperature $T$ for the state with energy $E$ is

$$
B(E)=\frac{1}{\exp (E / k T)-1}
$$

where $k$ is Boltzmann's constant (Eisberg 1961). $E$ is related to $\lambda$ by

$$
E=h v=h c / \lambda
$$

where $v$ is the frequency of the state. Substituting eq. (18) into (17), we obtain the probability density as a function of the wavelength $\lambda$

$$
P(\lambda)=\frac{1}{\exp (h c / k T \lambda)-1}
$$

Assuming that $\lambda \gg h c / k T$, by series expansion of the exponential function, and taking the first order approximation, we have

$P(\lambda)=\lambda_{1} P\left(\lambda_{1}\right) / \lambda$

where $\lambda_{1}=R_{\mathrm{o}}$. For gravitons in an elliptical orbit with average radius $R$, let their wavelength be $\lambda$, which being equal to their range or mean free path, equals the perimeter of the orbit.

$\lambda=2 \pi R$

Substituting eq. (21) into eq. (20), we obtain the probability density as a function of $R$,

$P(R)=R_{\mathrm{o}} P\left(R_{\mathrm{o}}\right) / R$

In terms of $P(R), \Delta \lambda(R)$ is defined as

$\Delta \lambda_{\mathrm{A}}(R)=\int_{R_{\mathrm{o}}}^{R} \lambda(R) P(R) d R / \int_{R_{\mathrm{o}}}^{R} P(R) d R$

Substituting eq. (21) and (22) into eq. (23), and evaluating the integrals, we get

$\Delta \lambda_{\mathrm{a}}(R)=\frac{R-R_{\mathrm{o}}}{c_{2}+\ln \left(R / R_{\mathrm{o}}\right)}$

where $c_{2}$ is a constant of integration. Substituting eq. (24) into eq. (16), we have

$\lambda_{\mathrm{A}}(R)=R_{\mathrm{o}}+\frac{R-R_{\mathrm{o}}}{c_{2}+\ln \left(R / R_{\mathrm{o}}\right)}$

Applying the condition of continuity of $\frac{d \lambda_{\mathrm{A}}(R)}{d R}$ at $R=R_{\mathrm{o}}$, it can be shown that $c_{2}=1$. Consequently if $R>R_{\mathrm{o}}$,

$\lambda_{\mathrm{A}}(R)=R_{\mathrm{o}}\left[1+\frac{\left(R / R_{\mathrm{o}}\right)-1}{1+\ln \left(R / R_{\mathrm{o}}\right)}\right]$

The condition of eq. (15.1), namely $\lambda_{\mathrm{A}}(R)=R$, is not satisfied by eq. (26), thus the region $R>R_{\mathrm{o}}$ is beyond the Newtonian region. Accordingly, the radius $R_{\mathrm{o}}$ separates the inner region from the outer region definitively; and the inner region $R \leq R_{\mathrm{o}}$ may be called the NGT region, while the outer region $R>R_{\mathrm{o}}$ may be called the QGT region. Moreover, from the term $\left(R / R_{\mathrm{o}}\right)$ in eq. (26), we may define $R_{\mathrm{o}}$ as the gravitational scale-length of a galaxy.

\subsection{The circular speed of a star in a spiral galaxy}

In Newtonian gravity, the gravitational potential $\Phi_{\mathrm{n}}(R)$ at a point $P$, which is at a radius $R$ from a central mass $M(R)$ at the origin $\mathrm{O}$, is

$\Phi_{\mathrm{n}}(R)=-\frac{G_{\mathrm{n}} M(R)}{R}$

where $G_{\mathrm{n}}$ is the Newtonian gravitational constant. For a star in an orbit of average radius $R$, its Newtonian circular speed is (Binney $\&$ Tremaine 2008)

$V_{\mathrm{n}}(R)=\sqrt{-\Phi_{\mathrm{n}}(R)}=\sqrt{\frac{G_{\mathrm{n}} M(R)}{R}}$ 
Analogously, applying eq. (6.2), the quantum circular speed is

$V_{\mathrm{q}}(R)=\sqrt{-\Phi_{\mathrm{q}}(R)}=\sqrt{\frac{G_{\mathrm{q}} M(R) \cosh \left[R / \lambda_{\mathrm{A}}(R)\right]}{R}}$

If $R \leq R_{\mathrm{o}}$, combining eq. (15.1), (28) and (29), we obtain

$V_{\mathrm{q}}(R)=V_{\mathrm{n}}(R)$

i.e. QGT reduces to NGT, as expected.

If $R>R_{\mathrm{o}}$, from eq. (11.1), (28) and (29), we have

$V_{\mathrm{q}}(R)=V_{\mathrm{n}}(R) \sqrt{\frac{\cosh \left[R / \lambda_{\mathrm{A}}(R)\right]}{\cosh (1)}}$

Eq. (31) implies that as $R$ increases beyond $R_{\mathrm{o}}$, the quantum speed becomes increasingly greater than the Newtonian speed, as a result of the cooperation of gravitons with antigravitons. From eq. (26) and (31), it can be shown that in the limit

$R \rightarrow \infty, V_{\mathrm{q}} \rightarrow V_{\mathrm{n}} \sqrt{\frac{R}{2 R_{\mathrm{o}} \cosh (1)}}$

\section{Comparing theory with the data of NGC6503}

The data of the rotation curve of the spiral galaxy NGC6503, in the Ph.D. thesis of Begeman (1987), were chosen for comparison with our quantum gravity theory (QGT). Lying in the Local Void (Finlay 2003), NGC6503 is an isolated galaxy, relatively free from gravitational disturbances from other galaxies.

\subsection{Mass distributions}

In analyzing the mass distributions of NGC6503, both the gaseous and stellar components were taken into account.

\subsubsection{Gas}

The radial distribution of HI surface density was obtained from the photometric data provided by Wevers et al. (1986). For improved accuracy, the surface density function is represented by a sum of two exponential functions, each with its own range, central extrapolated surface density $L_{\mathrm{o}}$, and scale-length $R_{\mathrm{d}}$, as shown in Table 1 . The rms deviation of the 25 photometric data points from the fitted function is 4.4 per cent.

Table 1: Photometry of Hydrogen Gas in NGC 6503

\begin{tabular}{lll}
\hline Range $(\operatorname{arcsec})$ & $L_{\mathrm{o}}\left(\mathrm{M}_{\mathrm{e}} \mathrm{pc}^{-2}\right)$ & $R_{\mathrm{d}}(\operatorname{arcsec})$ \\
\hline$R \leq 405.6$ & $6.063 \pm 0.016$ & $300.7 \pm 17.1$ \\
$R>405.6$ & $25.62 \pm 2.26$ & $145.4 \pm 12.8$ \\
\hline
\end{tabular}

Adopting a distance of 5.2 Mpc for NGC 6503 from the paper of Karachentsev \& Sharina (1997), and using an optically thin model, $M_{\text {gas }}(R)$, the mass of gas within a radius of $R$, was calculated as a function of $R$. Next, the total mass of the gas envelope was evaluated to be $(7.25 \pm 0.79) \times 10^{8} \mathrm{M}_{e}$, which agrees with the value of $7.0 \times 10^{8} \mathrm{M}_{e}$ given by Wevers et al. Then, the radius of the radial center of mass (RCM) of the gas envelope was computed to be $(730.6 \pm 49.5)$ arcsec. And the circular velocity $V_{\text {gas }}(R)$ curve due to the gas envelope, according to Newtonian gravity, is presented in the lower curve of Fig. 1.

\subsubsection{Stars}

For radii less than 134.5 arcsec, the red-band photometric data of the stars were obtained from Bottema (1989); and for larger radii, were converted from Wevers et al. (1986) by a formula derived by Bottema (1989), with an accuracy of better than 0.15 mag. For higher precision, the surface brightness function is represented by a sum of three exponential functions, each with its own range, central extrapolated surface brightness $L_{\mathrm{o}}$, and scale-length $R_{\mathrm{d}}$, as shown in Table 2. The standard deviation of the 67 photometric data points from the fitted function is 2.14 per cent.

Table 2: Red Band Photometry of the Stars in NGC6503

\begin{tabular}{lll}
\hline Range $(\operatorname{arcsec})$ & $L_{\mathrm{o}}\left(\mathrm{mag} \operatorname{arcsec}^{-2}\right)$ & $R_{\mathrm{d}}(\operatorname{arcsec})$ \\
\hline$<5.8$ & $17.93 \pm 0.04$ & $7.91 \pm 0.34$ \\
$5.8-193.5$ & $18.59 \pm 0.03$ & $44.79 \pm 0.46$ \\
$>193.5$ & $20.58 \pm 0.16$ & $77.62 \pm 1.40$ \\
\hline
\end{tabular}

The above data have not been corrected for the inclination of NGC 6503. According to Begeman (1987), the average inclination of NGC 6503 was $73.8^{\circ}$. Wevers et al. (1986) finished taking data on 1979 August 31, on which date the inclination of the Ecliptic was $23.21^{\circ}$, so the average angle between the galactic plane of NGC 6503 and the Ecliptic was $50.59^{\circ}$. The inclination factor, secant $\left(50.59^{\circ}\right)$, was used to obtain face-on values of the surface brightness. Having analyzed the surface brightness function into a sum of three exponential functions, by assuming that the mass-tolight $(M / L)$ ratio is constant for different brightness, an optically thin model was used to calculate $M_{\text {red }}(R)$, the mass distribution curve of the red-band stars. The purpose of using exponential functions is that their products with polynomial functions are directly integrable without resorting to numerical integration, hence ensuring higher mathematical accuracy in computing mass or the radial center of mass (RCM). Taking the inclination factor into account, the total mass of the red stars was computed to be $(2.416 \pm 0.091) \times 10^{10} \mathrm{M}_{e}$. The radius of the RCM of the red stars was calculated to be $(76.34 \pm 1.17)$ arcsec, which equals the radius of the RCM of all the stars, for a constant $M / L$ ratio.

\subsection{The Newtonian circular speed curve}

From Begeman's rotation curve of NGC 6503, it is estimated that the circular speed reaches a maximum when radius is $137.3 \mathrm{arcsec}$ For the first 5 data points, the radii were less than 125 arcsec, Newtonian gravity theory was tentatively assumed to be exact. Let $\Upsilon_{\text {red }}$ be the $M / L$ ratio for the red stars, which is related to the central mass $M(R)$ by

$M(R)=\Upsilon_{\text {red }} M_{\text {red }}(R)+M_{\text {gas }}(R)$

Using eq. (28) and (33), and the condition that the Newtonian circular speed agrees with the observed speed of the fifth point ( $R=125 \operatorname{arcsec}$ ), a first solution of $\Upsilon_{\text {red }}$ is 1.401 . Using an iterative procedure, a more precise value of $\Upsilon_{\text {red }}$ for generating the best-fitting curve of the whole range (25-775 arcsec) was determined to be 1.403 . Using this $M / L$ ratio, the mass of all the stars was calculated to be $(3.390 \pm 0.128) \times 10^{10} \mathrm{M}_{\mathrm{e}}$. Next, the Newtonian rotation curve due to stars, $V_{\text {stars }}(R)$, is presented in the upper curve of Fig. 1. Then, the Newtonian rotation curve $V_{n}(R)$ due to both stars and gas is shown in the lower curve of Fig. 3. 


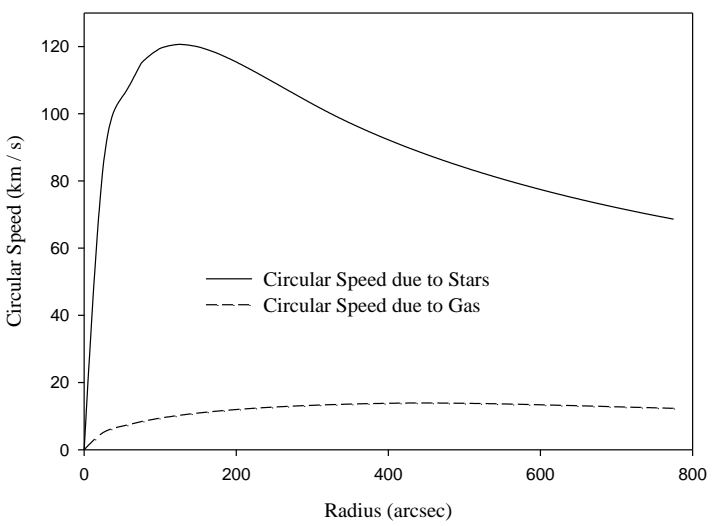

Fig.1: Rotation Curves Calculated from Photometric Data

Then the Newtonian rotation curve $V_{n}(R)$ due to both stars and gas, is shown in the lower curve of Fig. 3.

\subsection{The quantum circular speed curve}

From the above results of the masses of the gas envelope and all the stars, plus the radii of their radial centers of mass, the radius of the galactic RCM of NGC6503 was determined to be $(90.04 \pm 1.63)$ arcsec. Applying eq. (8), the gravitational scalelength $R_{\mathrm{o}}$ of the galaxy was evaluated to be (141.4 \pm 2.56$)$ arcsec, validating our earlier assumption in sec. 3.2 that radii less than 125 arcsec are within the Newtonian region. Using eq. (15.1) for $R \leq R_{\mathrm{o}}$, and eq. (26) for $R>R_{\mathrm{o}}$, the graviton wavelength $\lambda_{\mathrm{A}}(R)$ curve was calculated, and presented in Fig. 2. The best-fitting quantum circular speed $V_{q}(R)$ curve, with a $M / L$ ratio of 1.403 , was computed from eq. (31) and presented in the full-line curve of Fig. 3.

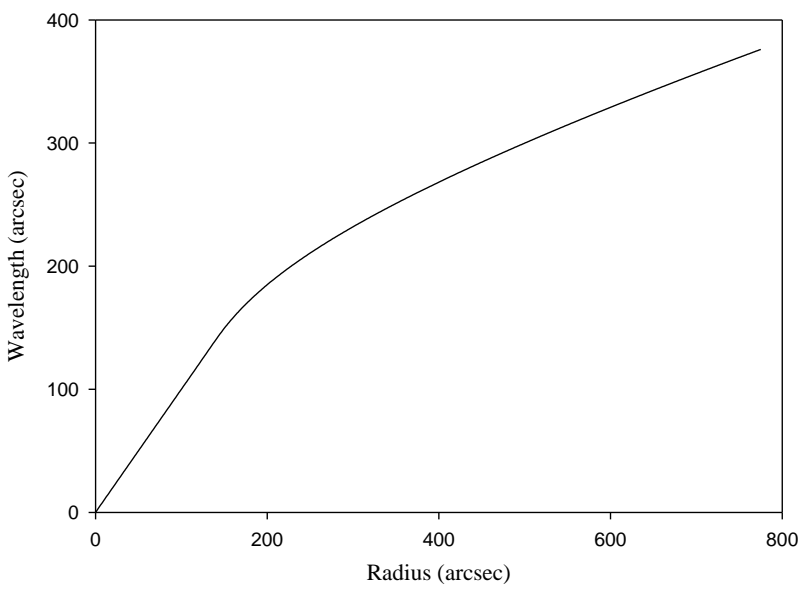

Fig. 2: Graviton Wavelength as a Function of Radius

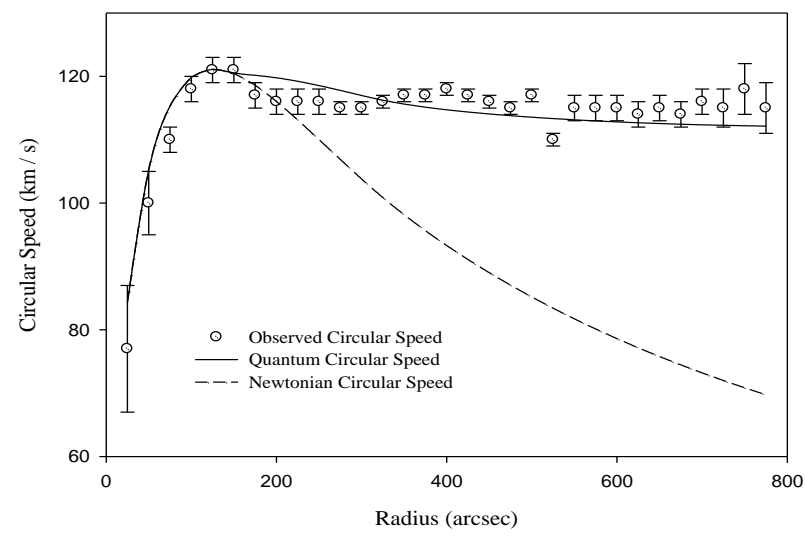

Fig. 3: Theory versus Data of NGC6503

\subsection{Verification of the quantum gravity theory}

In Fig. 3, the observed rotation curve of NGC6503 is compared with two theoretical curves, the quantum gravity curve and the Newtonian gravity curve. In the inner region $\left(R \leq R_{\mathrm{o}}=141.4\right.$ $\operatorname{arcsec}$ ), the quantum curve, which reduces to the Newtonian curve here, is in agreement with the observed circular speeds, within observational errors. After the observed rotation speed $V_{o b}$ reaches its maximum $(R=137.3 \mathrm{arcsec})$, it declines monotonically, though more and more gradually. In the outer region $\left(R>R_{\mathrm{o}}\right)$ the quantum gravity curve agrees with the measured speeds, and is increasingly better than the Newtonian gravity curve. For the entire experimental range of 25.0 to 775.0 arcsec, the standard deviation of the quantum circular speeds $V_{q}$ from the observed circular speeds $V_{o b}$ is 3.0 (2.99) per cent, within the rms error of the observed speeds, 3.0 (3.04) per cent. For an illustration of the quantitative difference between QGT and NGT in the outer region, the two theoretical curves were compared at the radii of 325,500 , and 750 arcsec. The percentage differences of the quantum speed $V_{q}$ from the Newtonian speed $V_{n}$ (with $V_{q}$ as denominator) at these radii are respectively 13.23, 25.00, 36.87 per cent.

\section{Discussions}

\subsection{The concept of negative mass}

In order to explain the concept of negative mass, let us consider a thought experiment. Let a gravitational force be applied simultaneously to a graviton with mass $m$ and an antigraviton with mass $-m$, the two accelerations will have the same magnitude but opposite directions.

\subsection{The non-annihilation of a graviton by an antigraviton}

Let a graviton be brought near to an antigraviton, they repel each other because the gravitational force between them is repulsive. So as they come near each other, they do not annihilate one another, unlike the electrical case of a positron and an electron.

\subsection{The Finite Wavelength of the Graviton}

The quarter fundamental wavelength of the gravitons of the galaxy NGC6503 was determined to be 141.5 arcsec. As the galaxy 
NGC6503 was at a distance of $5.2 \mathrm{Mpc}$, the fundamental wavelength $\lambda_{\mathrm{o}}$ of the gravitons was $14.27 \mathrm{kpc}$ or $4.402 \times 10^{17} \mathrm{~m}$

This result implies that the wavelength of the graviton is finite, contrary to the common belief that it is infinite.

\subsection{The non-zero rest mass of the graviton}

Using the relation $m=h / c \lambda$ for a relativistic particle, the expectation value of the dynamic mass of the gravitons in NGC6503 is computed to be $5.021 \times 10^{-53} \mathrm{~kg}$. This implies that the rest mass of the graviton must be non-zero, otherwise the dynamic mass would be exactly zero. This result contradicts the common idea that rest mass of the graviton is exactly zero.

\subsection{Virtual mass amplification by antigravitons}

If $R>R_{\mathrm{o}}$, from eq. (6.2), (15.1), and (27), the ratio of the quantum and Newtonian gravitational potentials can be written as

$\frac{\Phi_{\mathrm{q}}(R)}{\Phi_{\mathrm{n}}(R)}=\frac{M(R) \cosh [R / \lambda(R)] / \cosh (1)}{M(R)}$

By comparing the numerator and the denominator of the righthand side of eq. (34), it can be seen that the effect of the cooperation of antigravitons with gravitons is that the quantum potential is equivalent to a Newtonian potential, in which the central mass $M(R)$ has been multiplied by a factor of $\{\cosh [R / \lambda(R)] / \cosh (1)\}$. For several decades before the discovery of the antigravitons, this virtual mass-amplification effect had been misunderstood by supporters of the dark matter hypothesis as an evidence of the existence of dark matter.

\subsection{The Schwarzschild metric}

In order to illustrate the theoretical power of the quantum gravity theory (QGT), let us consider the Schwarzschild metric, as an example of showing how a formula in general relativity theory (GRT), which contains a gravitational potential term, may be revised by QGT. In the empty space outside a static point mass located at the origin, according to GRT, the metric equation at a point $\mathrm{P}$ with the spherical-polar coordinate $(R, \theta, \varphi)$ is:

$\mathrm{d} s^{2}=c^{2} \mathrm{~d} \tau^{2}=c^{2} \mathrm{~d} t^{2}\left(1-\frac{2 \Phi(R)}{c^{2}}\right)-\frac{\mathrm{d} R^{2}}{1-2 \Phi(R) / c^{2}}-R^{2} \mathrm{~d} \theta^{2}-R^{2} \sin ^{2} \theta \mathrm{d} \varphi^{2}$

where $\theta$ is the polar angle, $\varphi$ is the azimuth angle, $\mathrm{d} s$ is the line element, $\tau$ is the proper time, $t$ is the local time coordinate, and $\Phi(R)$ is the gravitational potential at P. Hence, for $i, \mathrm{j}=1-4$, the elements of the metric tensor are (Kenyon 1990):

If $i \neq j, g_{i j}=0$; and

$g_{11}=\left(1-\frac{2 \Phi(R)}{c^{2}}\right) ; g_{22}=-\left(1-\frac{2 \Phi(R)}{c^{2}}\right)^{-1}: g_{33}=-R^{2}: g_{44}=-R^{2} \sin ^{2} \theta$

In GRT, the gravitational potential $\Phi(R)$ in eq. (36) is equated with the Newtonian potential $\Phi_{\mathrm{n}}(R)$, as given by eq. (27). However, in QGT, $\Phi(R)$ is identified with the quantum potential $\Phi_{q}(R)$, as given by eq. (6.2). If eq. (6.2) is substituted into eq. (36), a GRT formula of the Schwarzschild metric tensor will be transformed into a quantum general relativity theory (QGRT) formula of the metric tensor outside a point mass.

\subsection{Gravitational time dilation}

To further demonstrate the theoretical ability of QGT, let us consider gravitational time dilation, as an example of showing how a GRT formula, which contains gravitational potential terms, may be amended by QGT. Let the gravitational potential at two points $P_{1}$ and $P_{2}$ be $\Phi_{1}$ and $\Phi_{2}$ respectively, and let the rates of two clocks placed at $\mathrm{P}_{1}$ and $\mathrm{P}_{2}$ be $f_{1}$ and $f_{2}$ respectively. According to GRT, the ratio of the clock rates is (Rindler 1977)

$\frac{f_{2}}{f_{1}}=\exp \left[\left(\Phi_{1}-\Phi_{2}\right) / c^{2}\right]$

In GRT, the two gravitational potential terms $\Phi_{1}$ and $\Phi_{2}$ in eq. (37) are equated with Newtonian potentials; in QGT, the gravitational potential terms are identified with the quantum potentials. In particular, if $\mathrm{P}_{1}$ and $\mathrm{P}_{2}$ are two points at radii $R_{1}$ and $R_{2}$ from the center of a galaxy, by using eq. (6.2) twice, we obtain

$\frac{f_{2}}{f_{1}}=\exp \left\{\frac{G_{\mathrm{q}}}{c^{2}}\left[\frac{M\left(R_{2}\right) \cosh \left[R_{2} / \lambda_{\mathrm{a}}\left(R_{2}\right)\right]}{R_{2}}-\frac{M\left(R_{\mathrm{1}}\right) \cosh \left[R_{\mathrm{1}} / \lambda_{\mathrm{a}}\left(R_{\mathrm{1}}\right)\right]}{R_{1}}\right]\right\}$

In this way, a GRT formula of gravitational time dilation has been upgraded into a QGRT formula.

\section{Conclusions}

The main results of this paper may be summarized as follows.

1) Using special relativity theory, it was discovered that to each graviton with mass $m$ and wavelength $\lambda$, there coexists an antigraviton with mass $-m$ and wavelength $-\lambda$.

2) The graviton has a finite wavelength, and a non-zero rest mass As a corollary, the antigraviton has a finite, negative wavelength, and a non-zero, negative rest mass.

3) From the discovery of the antigraviton, by applying quantum theory, a new quantum gravity theory (QGT) has been derived providing two core formulas: one for the gravitational potential, and another for the graviton wavelength, as functions of the radius $R$.

4) From these two core formulas, formulas for related dynamical variables may be derived. For example, gravitational acceleration can be calculated as the gradient of the gravitational potential. In this paper, formulas of the circular speed, the Schwarzschild metric tensor, and gravitational time dilation have been presented.

5) The gravitational scale-length $R_{\mathrm{o}}$ of a spiral galaxy is related to its mass distribution, and is equal to $0.5 \pi(\approx 1.5708)$ times the radius of its radial center of mass.

6) Newtonian gravity theory (NGT) is partially true. If the radius $R \leq R_{\mathrm{o}}$, it is exact, as QGT reduces to it; if $R>R_{\mathrm{o}}$, NGT is inadequate, and has been superseded by QGT. The inner region $R \leq R_{\mathrm{o}}$ may be called the NGT region, while the outer region $R>R_{\mathrm{o}}$ may be called the QGT region.

7) The Newtonian gravitational constant $G_{\mathrm{n}}$ is applicable only in the NGT region, but the newly discovered quantum gravitational constant $G_{\mathrm{q}}$ is applicable universally, and $G_{\mathrm{q}}=0.6480543 G_{\mathrm{n}}(7$ sig. fig.).

8) When the QGT was compared with the data of the rotation curve of the galaxy NGC 6503, the theory was verified within the standard error of the observed rotation speeds, 3.0 per cent. Checking how well the QGT is supported by the data of other spiral galaxies (e.g. NGC2403, NGC3198 etc.) is a good project for further study. 
9) In the QGT region of a galaxy, an antigravity effect due to the antigravitons is a virtual amplification of the central mass. Before the discovery of the antigravitons, this effect has been misunderstood by many for about four decades, as an evidence of the existence of dark matter in galaxies.

10) Our new QGT was solidly built on two fundamental pillars of modern physics, viz. relativity theory (RT) and quantum theory (QT). Our motivation is not to challenge the pillars of modern physics but it is a clarification of logical implications of these two pillars.

11) With two concrete examples, it has been demonstrated that the QGT can be used to revise formulas of general relativity theory, and transform them into those of a quantum general relativity theory.

12) Since the new QGT was developed without the ad hoc dark matter hypothesis (DMH), the successful verification of the QGT implies that the DMH is unnecessary in galaxy dynamics. It is suggested the so-called "dark matter" problem can be solved by relinquishing the DMH and adopting our new QGT, or antigraviton-graviton theory. It seems that this theory would become a significant development in gravitational physics.

\section{Acknowledgement}

We are grateful to the anonymous referees for their valuable comments. We also thank the comments from Prof. Philip Mannheim and Prof. Pavel Kroupa during the preparation of this paper. The encouragement from Wai-Chuen Leung and WingChin Wong are hereby gratefully acknowledged.

\section{References}

[1] Begeman K (1987), PhD thesis, Groningen State University, p. 51.

[2] Binney J \& Tremaine S (2008), Galactic Dynamics, Princeton University Press, Princeton, NJ , p. 62

[3] Bottema R (1989), "The Stella Velocity Dispersion of the Spiral Galaxies NGC6503 and NGC6340", Astronomy and Astrophysics, Vol. 221, No.2, 236-249.

[4] Eisberg RM (1961), Fundamentals of Modern Physics, John Wiley, New York, p. 389.

[5] Finlay WH (2003), Concise Catalog of Deep-Sky Objects, Springer, London, p. 204.

[6] Forshaw JR \& Smith AG (2009), Dynamics and Relativity, John Wiley, Chichester, p.149.

[7] Karachentsev ID \& Sharina ME (1997), "Distance to the Spiral Galaxy NGC6503 Situated in the Local Void", Astronomy and Astrophysics, Vol. 324, 457-460.

[8] Kenyon IR (1990), General Relativity, Oxford University Press, Oxford, p. 44.

[9] Kent SM (1987), "Dark Matter in Spiral Galaxies. II. Galaxies with HI Rotation Curves", The Astronomical Journal, Vol.93 No.4, 816-832.

[10]Rindler W (1977), Essential Relativity, Springer, New York, p. 118.

[11]Rubin VC (1983), "Dark Matter in Spiral Galaxies", Scientific American, Vol.248, No.6, 88-98,101.

[12]Van Albada TS, Bahcall JN, Begeman K \& Sancisi R (1985) "Distribution of Dark Matter in the Spiral Galaxy NGC3198", The Astrophysical Journal, Vol.295, 305-313.

[13] Van Albada TS \& Sancisi R (1986), "Dark Matter in Spiral Galaxies", Philosophical Transections of the Royal Society of London. Vol. A 320, 447-464

[14]Wevers BMHR, Van Der Kruit PC \& Allen RJ (1986), "The PalomarWesterborks Survey of Northern Spiral Galaxies", Astronomy \& Astrophysics Supplement Series, Vol. 66, 505-662.

[15]Yukawa H (1935), "On the Interaction of Elementary Particles. I.", Proceedings of the Physico-Mathematical Society of Japan, Vol.17, 27-36. 